\title{
Electron-beam enhanced fusion of binary metals and alloying in melts
}

\author{
T VIJAYAN and V K ROHATGI \\ Plasma Physics Division, Bhabha Atomic Research Centre, Trombay, Bombay 400085, India \\ MS received 27 January 1984; revised 27 July 1984
}

\begin{abstract}
Binary mixing and alloying during electron-beam fusion in bi-metal joints have been investigated and presented in this paper. In case of beam-power densities greater than about $10^{10} \mathrm{Wm}^{-2}$, rapid fusion of metals is accompanied by violent agitation of the melts. The resulting mass transport in the melt is described here from an enhanced mass diffusion coefficient $D=D_{s}\left(W^{\prime} / a_{0} \eta\right)^{1 / 2}$, where $D_{3}$ is mass diffusivity in solid, $W^{\prime}$ is beam-power density, $a_{0}$ is acceleration in melt growth and $\eta$ is the coefficient of viscosity. Moreover, a uniformly mixed central-width $w^{\prime}$ is predicted in the fusion zone (total width $w$ ), where, $w^{\prime}$ is significantly dependent on $\eta$ and $w$ through $W^{\prime}$. For these conditions, the continuity of mass transport in stationary and travel modes of the source has been solved using line and plane source models and conservation principles. The predictions from these calculations have been validated through quantitative analyses of experiments.
\end{abstract}

Keywords. Electron-beam fusion; dissimilar metals; vapour-pressure kinetics; alloying; diffusion.

\section{Introduction}

Significant fusion and deep penetration in thick and dissimilar metals are attained using high-density electron beams as the heat source. In this case, the energy dissipation by beam into metal through cavity formation in melt, is fairly well understood (Andrews \& Atthey 1976; Klemens 1976; Vijayan \& Rohatgi 1983). Moreover, as far as mass transport in melts is concerned, there are indications from the present as well as other experiments (Miyazaki \& Taniguchi 1972; Rykalin et al 1978; Schwarz 1964; Weber 1972) that melt agitation and flow are present during the beam-fusion process. Such conditions are expected to give rise to rapid melt mixing. Under these conditions, metals which are otherwise incompatible can be fused together. However, the properties of the joint in this case, in general, will be characterised by the composition and the distribution of the constituents in the joint region as well as the compatibility of the two metals for alloying. In this context, an understanding of the mass transport and mixing in melts of binary metal combinations, which are fused by an electron beam, is necessary. Investigations on these lines are of considerable importance in dissimilarmetal joining research. Hence, studies on these aspects have been carried out. In the present study, physical descriptions of beam fusion in dissimilar metals, mass transport and intermixing of binary melts, and theoretical models for these have been developed. The results of the calculations have been validated through experiments.

A list of symbols appears at the end of the paper. 


\section{Fusion behaviour in dissimilar metals}

Fusion of metals $A$ and $B$ which form a binary system, will depend on their heat contents for melting per unit volume, on the absorption coefficients $\left(\alpha_{A}\right.$ and $\left.\alpha_{B}\right)$, and on their coefficients of thermal conductivities $\left(K_{A}\right.$ and $\left.K_{B}\right)$. In case of significant differences in these values, while the beam input sharing is equal, fusion in the two metals will not be uniform, whereas for actual joining of the metals it is desired that this be nearly identical. However, the heat for fusion growth (Vijayan 1982) increases with the square root of the coefficient of thermal conductivity. Taking this into consideration and including the absorption coefficients, the ratio of the heat inputs $\left(Q_{A} / Q_{B}\right)$ needed for equal fusion in semi-infinite targets of bi-metals, is obtained as

$$
Q_{A} / Q_{B}=\delta \frac{\alpha_{B}}{\alpha_{A}}\left[K_{A} / K_{B}\right]^{1 / 2}
$$

where

$$
\delta=\frac{\rho_{A}\left(c_{A} T_{A}+L_{A}\right)}{\rho_{B}\left(c_{B} T_{B}+L_{B}\right)} .
$$

In general, (1) ideally fits the case of fusion depths of a few millimetres or more. In the case of deep-penetration fusion by an electron beam, the beam deposition (Klemens 1976; Rykalin et al 1978) takes place at the base of a key hole in the melt, and hence, the absorption of beam is expected to be nearly total. For this case, (1) is modified as

$$
Q_{A} / Q_{B}=\delta\left[K_{A} / K_{B}\right]^{1 / 2} \text {. }
$$

In metal diaphragms of very small thickness $(<0.1 \mathrm{~mm})$, heat losses due to thermal conduction in the bulk are negligible, and (1) for the same has been modified as

$$
Q_{A} / Q_{B}=\delta\left(\alpha_{B} / \alpha_{A}\right)
$$

Equation (4) is valid for thin diaphragms in general, regardless of the width. In case of semi-infinite fusion depth $h$ and for plate widths nearly equal to the melt widths, the thermal conduction losses are minimum, and hence $Q_{A} / Q_{B}=\delta$. For plate widths between this and a semi-infinite width, $Q_{A} / Q_{B}$ will lie somewhere between $\delta$ and the value given by (3).

\section{Physical description of melt-mixing}

In case of electron-beam fusion of moderate depths (about a few $\mathrm{mm}$ ), the vapour pressure (Rykalin et al 1978) is expected to dominate the formation of a key hole in the melt. In a binary-metal combination $(A, B)$ to be fused in which metal $B$ has a higher vapour pressure than metal $A$, metal $B$ satisfies the pressure requirements towards the formation of a key hole at a lower temperature than metal $A$. However, on fusion of both the metals, the maximum temperature (at the key-hole base) in the melt is expected to be higher than the melting point $T_{A}$ of metal $A$. A binary system of this nature is illustrated in figure $1(\mathrm{a})$. In this example, the given metals are assumed to have extreme differences in their thermo-physical properties, where metal $\boldsymbol{A}$ needs a higher heat content for melting than metal $B$, due to higher values for the melting point, density, etc. In view of this, on normal incidence and arbitrary power sharing of electron beams on 


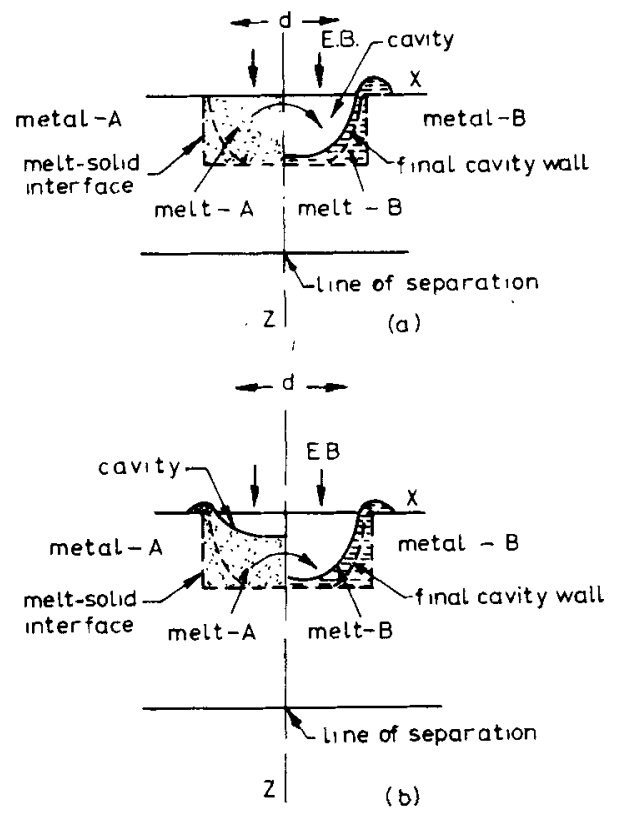

Figure 1. Models of binary fusion and mixing (a) in the case of extreme nature of thermophysical properties of metals, (b) in the case of metals having similar heating properties but different surface tension properties

the metals, metal $A$ melts after metal $B$ with a time lag. In this time, metal $B$ has meited and a partial cavity has already formed, and hence, as soon as metal $A$ melts it slides into the cavity in $B$. In this process, a part of melt $B$ gets submerged below melt $A$. This, on continued-beam heating, creates high vapour pressure conditions due to $B$ within, which consequently results in violent disturbances in the melt. In this process, a cavity, which satisfies the combined surface tension effects of both the melts, is formed. Also, as the vapour pressure inside the cavity increases further, the cavity base develops upto the melt base. Thereafter, the beam dissipates heat into a new depth in the solid and the chain of events, as described above, repeats in the new zone before proceeding to the next zone, and so on. In this manner, the two metals are fused and the melts are mixed in a continuous manner.

Melt behaviour in a binary system for equal power sharing in metals having similar fusion properties but extreme values for the surface tension is illustrated in figure 1(b). In this system, melting of the two metals takes place more or less simultaneously, but the cavities in the two melts tend to form with different radii. Therefore, sliding of one melt into the cavity in the other occurs and an equilibrium cavity is eventually formed. Further fusion and mixing of the melts progress in a manner similar to the one described above. In case of metal properties between the two extremes explained here, and for different degrees of power sharing, the melt conditions can be obtained by suitably modifying the above models. In addition, temperature gradients induce convection currents in the melt. Also, as the cavity develops into a key hole, the melt is drawn up (Rykalin et al 1978) along the key hole wall due to pressure gradients. The melt-solid interface, on the other hand, tends to retard the melt flow in this case. The melt behaviour described above gives a fairly complete picture of mixing in the 
stationary source case. In case of transverse travel of source, in addition to these, the melt is drawn from the key hole front to the back, around the hole (Klemens 1976). Hence, through all these mechanisms the fused zone is rapidly mixed and is eventually made up of a liquid solution of the given composition and distribution.

\section{Theoretical formulation}

Beam fusion in metals, especially in case of deep penetration, gives rise to a number of nonlinearities in the heat and mass transfer events (Klemens 1976; Rykalin et al 1978) due to a continuously shifting beam-impact plane, extreme temperature-gradients, rapid vaporization, vapour pressure kinetics, etc. As a matter of fact, an exact solution of mass transport in the present problem is difficult. Therefore, the mass transfer for this case has been obtained presently in an approximate model. In general, deep penetration fusion by the electron beam, is described (Klemens 1976; Rykalin et al 1978) by a line source concept where concentration gradients in the central region of fusion in the beam direction can be neglected for all practical purposes. However, mass distributions in the $x$ - and $y$-directions will be significant. Moreover, a high beam density means extremely vigorous melting and melt agitation. Hence, for these turbulent conditions in the melt, the coefficient of mass diffusion $D$ is of an enhanced level. A semi-empirical expression for $D$ under these conditions has been obtained in the present study by analying the experimentally measured profiles of the mass concentrations inside the beam-fused welds of various bi-metal combinations, and fitting them to the theory. This expression is written as,

$$
D=D_{s}\left[W^{\prime} / a_{0} \eta_{T}\right]^{1 / 2} \text {. }
$$

In the present problem, mass transfer commences with full concentrations $C_{0 A}$ and $C_{O B}$ of the two metals $(A, B)$ on either side of the discontinuity. That is, the initial boundary conditions in this case, are

$$
\begin{aligned}
& \text { Metal } A \\
& \mathrm{~d} C_{A} / \mathrm{d} x=0, \mathrm{~d} C_{A} / \mathrm{d} y=0 \\
& \mathrm{~d} C_{A} / \mathrm{d} z=0, C_{B}=0, \\
& T=T_{0}, C_{A}=C_{0 A},
\end{aligned}
$$

Discontinuity

$$
\begin{aligned}
& \text { Metal } B \\
& \mathrm{~d} C_{B} / \mathrm{d} x=0, \mathrm{~d} C_{B} / \mathrm{d} y=0, \\
& \mathrm{~d} C_{B} / \mathrm{d} z=0, C_{A}=0, \\
& T=T_{0}, C_{B}=C_{0 B} .
\end{aligned}
$$

\begin{tabular}{|c|c|c|}
\hline \multirow[t]{2}{*}{ Metal $A$} & $\begin{array}{c}\text { Binary } \\
(A+B)\end{array}$ & Metal B \\
\hline & $=T_{A}$ & $=T_{B}$ \\
\hline $\begin{array}{l}\mathrm{d} C_{A} / \mathrm{d} x=0, \mathrm{~d} C_{A} / \mathrm{d} y=0 \\
\mathrm{~d} C_{A} / \mathrm{d} z=0, C_{B}=0 \\
T>T_{0}, C_{A}=C_{0 A}\end{array}$ & $\begin{array}{l}\mathrm{d} C_{A} / \mathrm{d} x \geqslant 0, \mathrm{~d} C_{B} / \mathrm{d} x \geqslant 0 \\
\mathrm{~d} C_{A} / \mathrm{d} y \geqslant 0, \mathrm{~d} C_{B} / \mathrm{d} y \geqslant 0 \\
\mathrm{~d} C_{A} / \mathrm{d} z \simeq 0, \mathrm{~d} C_{B} / \mathrm{d} z \simeq 0 \\
C_{0 A}>C_{A}>0 \\
0 \quad<C_{B}<C_{0 B} \\
T \quad \geqslant T_{A}, T_{B}\end{array}$ & $\begin{array}{l}\mathrm{d} C_{B} / \mathrm{d} x=0, \mathrm{~d} C_{B} / \mathrm{d} y=0 \\
\mathrm{~d} C_{B} / \mathrm{d} z=0, C_{A}=0 \\
T>T_{0}, C_{B}=C_{0 B} \\
\text { Interface }\end{array}$ \\
\hline
\end{tabular}

On beam irradiation, as fusion and mass transfer progress, concentrations of the solvent melt decrease, while those of the solute increase. The resulting conditions, in general, will be: 
Assuming $D$ as an invariant in the present problem the transient concentration of the element $A$, (i.e. $C_{A}$ ) is obtained (Crank 1964) as

$$
C_{A}=\frac{M_{l}}{4 \pi D_{A} t} \exp \left\{-\left[\left(x-x_{0}\right)^{2}+\left(y-y_{0}\right)^{2}\right] / 4 D_{A} t\right\} \text {. }
$$

Also, $C_{A}$ has been written here in terms of a concentration $C_{A 1}$ at a neighbouring point at $(\Delta x, \Delta y)$

$$
C_{A}=C_{A 1} \pm\left[\Delta x^{2}\left(\mathrm{~d} C_{A} / \mathrm{d} x\right)^{2}+\Delta y^{2}\left(\mathrm{~d} C_{A} / \mathrm{d} y\right)^{2}\right]^{1 / 2}
$$

In this equation, the concentration gradient $\mathrm{d} C_{A} / \mathrm{d} x$ is given as

$$
\frac{\mathrm{d} C_{A}}{\mathrm{~d} x}=\frac{-M_{l}\left[\left(x-x_{0}\right)^{2}+\left(y-y_{0}\right)^{2}\right]}{8 \pi D_{A}^{2} t^{2}\left(x-x_{0}\right)} \exp \left\{-\left[\left(x-x_{0}\right)^{2}+\left(y-y_{0}\right)^{2}\right] / 4 D_{A} t\right\} \text {. }
$$

Similarly $\mathrm{d} C_{A} / \mathrm{d} y, \mathrm{~d} C_{B} / \mathrm{d} x$ and $\mathrm{d} C_{B} / \mathrm{d} y$ have been obtained.

But in case of target travel (or source travel) in the $y$-direction as in welding, there will be a plane column of melt in this direction (Vijayan 1982) left behind by the beam, and which is yet to solidify. In this case concentration gradients in the $y$-direction (in (7) and (9)) also can be neglected. Further mass transfer in these regions will be taking place in the $x$-direction through convection. The transient concentrations and their gradients in this case are obtained as (Crank 1964)

$$
C_{A}=\frac{M_{p}}{2\left(\pi D_{A} t\right)^{1 / 2}} \exp \left[-\left(x-x_{0}\right)^{2} / 4 D_{A} t\right]
$$

where $M_{p}$ is the plane source strength per unit area and

$$
\frac{\mathrm{d} C_{A}}{\mathrm{~d} x}=\frac{-M_{p}\left(x-x_{0}\right)}{4 \pi^{1 / 2}\left(D_{A} t\right)^{3 / 2}} \exp \left[-\left(x-x_{0}\right)^{2} / 4 D_{A} t\right] .
$$

\section{Computations of mass transfer}

Mass transport in a binary melt formed by electron-beam fusion, is solved in this study through the following approximations and using the computation scheme given in figure 2. In electron-beam fusion, as stated earlier, a high density of the beam $\left(\sim 10^{10} \mathrm{Wm}^{-2}\right)$ means an extremely localised fusion and vigorous agitations in the melt. As a result, a uniformly mixed central region in the melt is observed in the experiments. The width $w^{\prime}$ of this region is obtained here by fitting these observations to the theory as

$$
w^{\prime}=w\left(\eta_{A}-\eta_{B}\right) / 2 \bar{\eta} .
$$

Here, $w$ and fusion depth $h$ have been obtained by solving for fusion growths (Vijayan 1982 ) in the given metals. And as $w$ increases (i.e. as beam power increases), $w^{\prime}$ also increases till $w=w_{0}$. For $w>w_{0}, w^{\prime}$ tends to decrease with $w$. This is in view of a less vigorous stirring in the melt provided by a lower density of beam. For this condition, $w^{\prime}$ is obtained as

$$
w^{\prime}=\frac{w_{0}^{3}}{w^{2}}\left(\eta_{A}-\eta_{B}\right) / 2 \bar{\eta}
$$




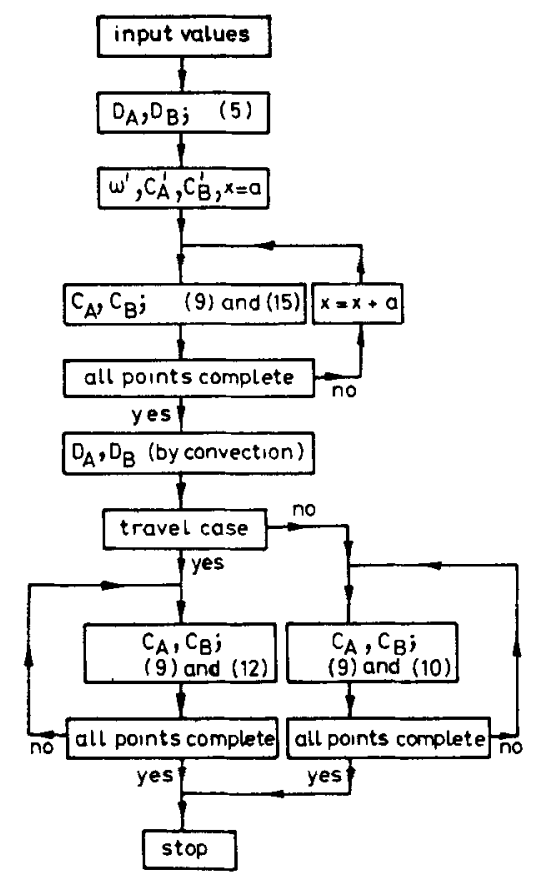

Figure 2. Flow chart of computation scheme (numbers in brackets refer to the equations concerned)

In the case of deep penetration, $w^{\prime}$ is. given by (13). The concentrations $C_{A}^{\prime}$ and $C_{B}^{\prime}$ of elements $A$ and $B$ inside $w^{\prime}$ are deduced from melt quantities $q_{A}$ and $q_{B}$ as $C_{A}^{\prime}=\left[q_{A} /\left(q_{A}+q_{B}\right)\right]$ and $C_{B}^{\prime}=\left[q_{B} /\left(q_{A}+q_{B}\right)\right]$. Concentrations surrounding $w^{\prime}$ are determined through the application of (9) and (10). For the interval of beam fusion, (10) is discretised as

$$
\frac{\mathrm{d} C_{A}}{\mathrm{~d} x}=\frac{-C_{A}^{\prime} a^{2} b\left[\left(x-x_{0}\right)^{2}+\left(y-y_{0}\right)^{2}\right]}{8 \pi b_{0} D_{A}^{2} t^{2}\left(x-x_{0}\right)} \quad \exp \left\{-b_{0}\left[\left(x-x_{0}\right)^{2}+\left(y-y_{0}\right)^{2}\right] / 4 D_{A} t\right\},
$$

Similarly $\mathrm{d} C_{A} / \mathrm{d} y$ and the gradients of $B$ can be written down.

In (15) $t$ is the beam-on-time $\left(t_{b}\right), a$ is used to normalise the source strength in conditions of beam, $b_{0}$ includes the effect of a small time duration of beam fusion and $b$ the effect of travel speed conditions, where $b$ is equal to $t$ in magnitude. $a=10^{-5} \mathrm{~m}$ and $b_{0}=10^{-4}$ are found from experiments of deep-penetration fusion. In case of source travel with speed $V, t$ equals $t_{b}=d / V$, where $d$ is the beam-spot width. In electron-beam welding, travel speeds in the region of 0.005 to $0.5 \mathrm{~m} / \mathrm{s}$ are of interest, and hence the $t_{b}$ value in this case will approximately be in the range of 0.1 to $0.001 \mathrm{~s}$.

Apart from these, for evaluating the mass transfer which takes place through convection subsequent to beam fusion when the beam is withdrawn from the point, the time $t_{1}$ required for the solidification of the melt has to be determined. This, in the line source case, is equal to

$$
t_{1}=\frac{\left(q_{m}-q\right) t_{b}}{q\left(K / K_{0}\right)^{1 / 2}} \frac{w}{d}
$$


where $q=\left[\rho\left(c T_{m}+L_{m}\right)\right]$; and $q_{m}=\left[\rho\left(c T+L_{m}\right)\right] ;$ Using $t_{1},(10)$ has been solved along with (9) to obtain the mass concentrations due to convection in the line source, where concentrations resulting from calculations during beam fusion are taken as the initial conditions. Hence, $M_{l}$ in (10) is equal to $C_{0} a^{2}$ where $C_{0}$ is average local concentration, $D_{A}$ is the coefficient of convective mass transfer (Cottrell 1964; Geiger \& Poirier 1973), distance step for differentiation is about $a$, and $t=t_{1}$ which is typically a fraction of a second or less for the deep penetration case.

In case of convection in a plane melt column as in a target travel case in welding, (12) and (9) have been solved subsequent to beam fusion. In this case, $M_{p}$ is equal to $C_{0} a$, and $t$ is time $\left(t_{2}\right)$ for the plane-melt column to solidify, where $t_{2}$ is obtained as

$$
t_{2}=t_{1}\left(y_{1} / w\right)
$$

with $y_{1}$ the length of the melt column left behind by the beam that is given as

$$
y_{1}=w\left(V_{0} K_{0} / V K\right)^{1 / 2}
$$

where $V_{0}$ is the travel speed at which $y_{1}=w$. In case of deep penetration, $t_{2}$ is usually about a second or so.

The present calculations thus consider the intermixing of melts during beam fusion in a line source, and further mass transports in melt through convection-in the line source in case of a stationary beam and target, or in plane column for the case of travel conditions in welding.

\section{Results and discussions}

The beam power fractions to give uniform fusion in different binary systems as calculated from (1)-(4) are tabulated in table 1. For arriving at these values, in case of metals having thermal conductivity $K<K_{0}, K_{A}$ or $K_{B}$ has been replaced by $K_{0}$ in these equations, whereas for metals where $K>K_{0}$, the same value has been used. In table 1 , it can be seen that the average temperatures in the melt increase with fusion depth $h$. This is in view of the temperature at the key-hole base (Vijayan 1982) being higher for larger $h$. Moreover, in a combination consisting of a poor thermal conductor and a good thermal conductor, in which the latter has a lower heat content for melting, the power sharing needed for uniform fusion shows a marked trend to reversal as $h$ increases. This is due to larger heat losses in bulk in a good conductor with increase in $h$. For example, in table 1 for $\mathrm{Fe}-\mathrm{Cu}$ thin foils, the fractional sharings are $0.62(\mathrm{Fe})$ and $0.38(\mathrm{Cu})$, while for $h \approx 50 \mathrm{~mm}$, the fractions are $0.39(\mathrm{Fe})$ and $0.61(\mathrm{Cu})$.

The mass-transport coefficients $D$ calculated for the various cases are given in table 2 ; in which the coefficients of viscosity $(\bar{\eta})$ are at the average temperatures given in table 1. The $D$ values given in column (4) of table 2 are for the convection case by assuming suitable temperature gradients between the melting points. The activation energy $E_{A}$ (tens of $\mathrm{kJ} / \mathrm{mol}$ ) (Vijayan 1982) used in the calculation of $D$ and in the corresponding frequency factor $D_{0}$ (Cottrell 1964; Geiger \& Poirier 1973) is at the boiling point. $D$ values given in the last column of table 2 are for the assumption of the temperature at the key hole as the maximum in the melt, the minimum being the lower value of the two melting points. It can be seen from here that even under such extreme temperature gradients, $D$ due to convection is only about $10^{-9} \mathrm{~m}^{2} / \mathrm{s}$ or less. This cannot adequately 


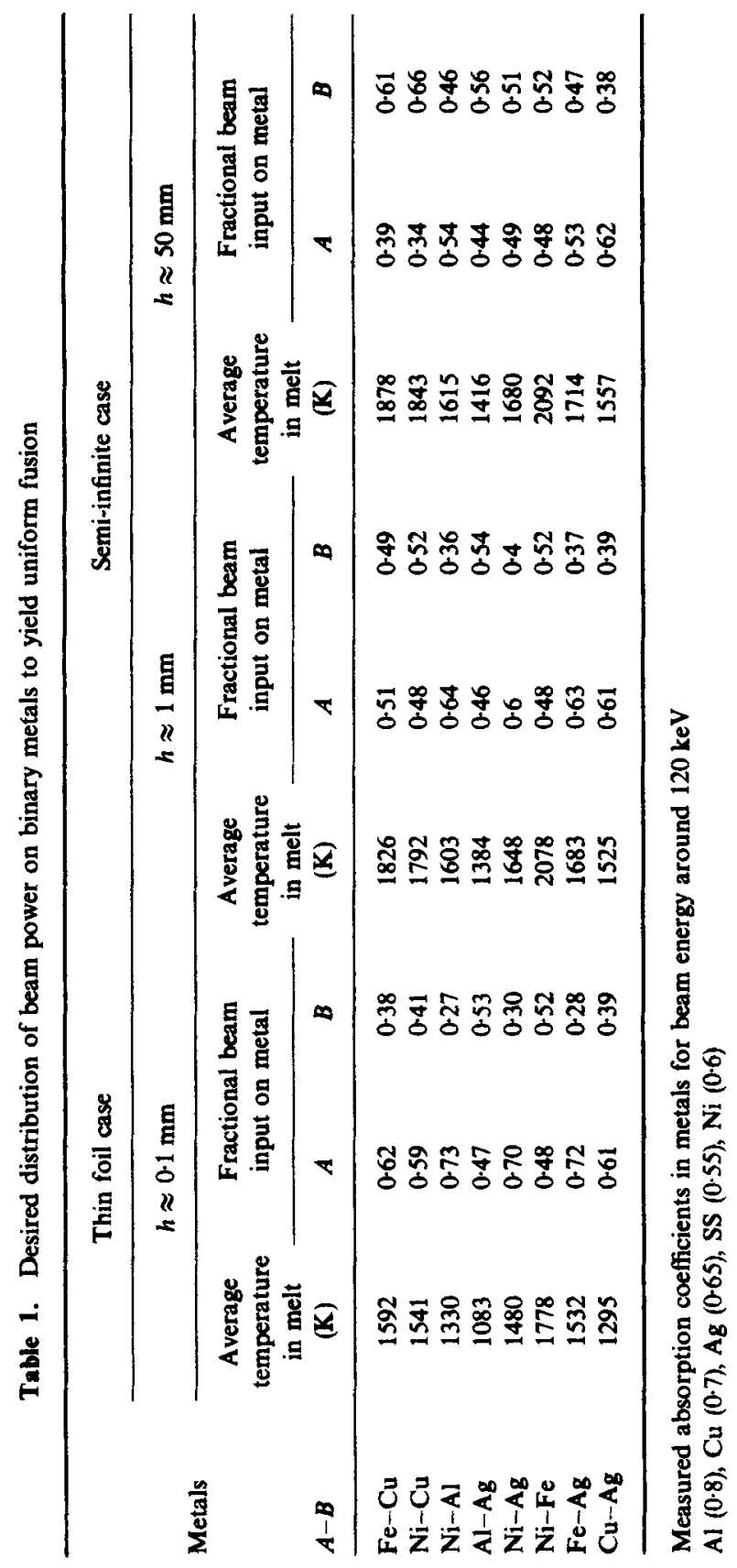




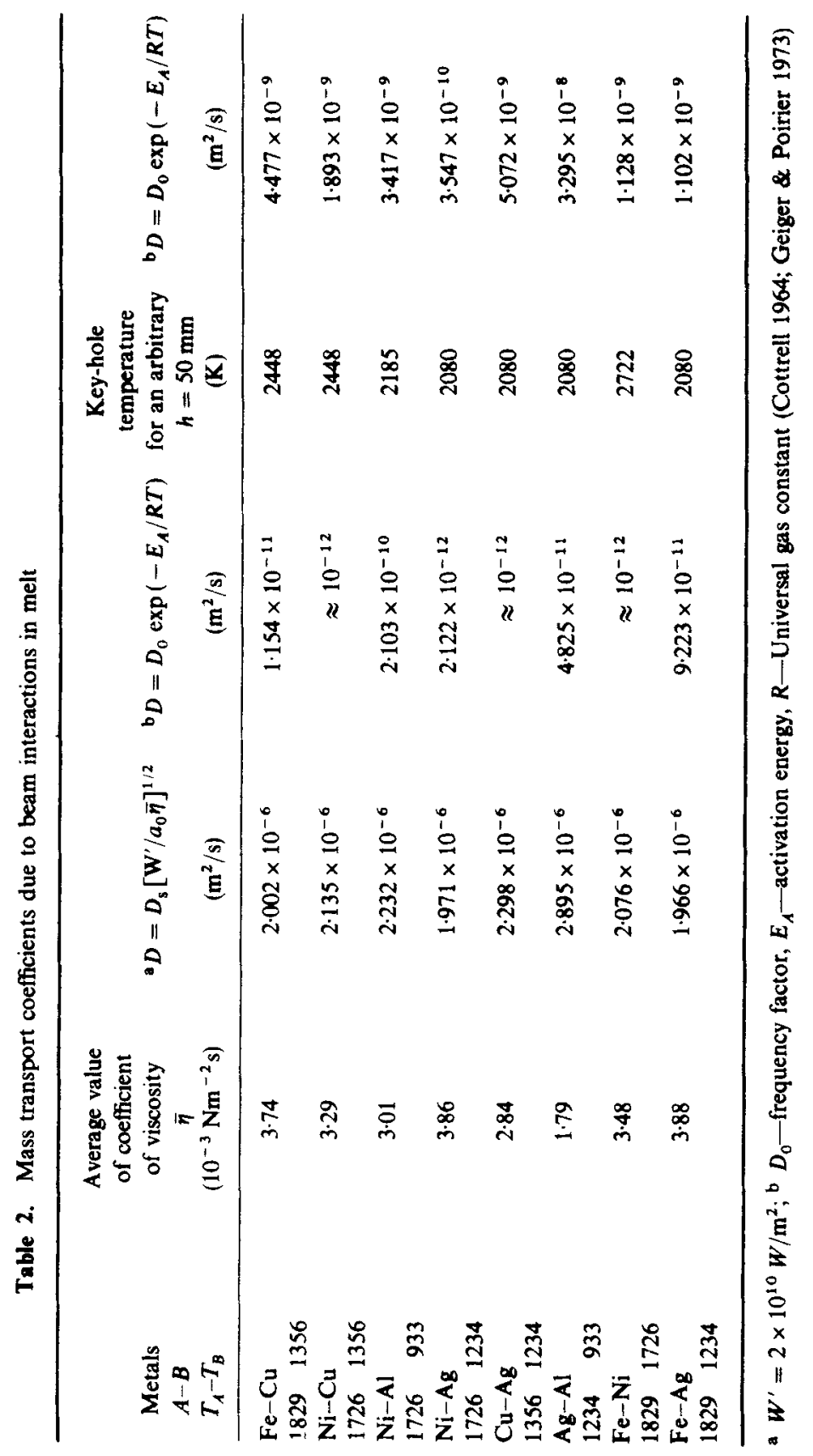


account for the high levels of mass transport taking place during beam fusion. On the other hand, $D$ values obtained using (5) and given in column (3) of table 2 are higher than $10^{-6} \mathrm{~m}^{2} / \mathrm{s}$ and are consistent with the type of mass transfer encountered here.

Using the computation scheme in $\S 5$ and $D$ values from table 2 , mass concentrations and distributions in the $x-z$ plane of the fusion zone for the source-travel case in various metal combinations have been determined. The results so obtained in this manner are plotted in figure 3 for a metal combination ( $\mathrm{Al}-\mathrm{Ag}$ ) having almost similar thermal properties (melt-mixing behaviour close to figure 1(b)). In this case, the results are at a given fusion width at level $A-A^{\prime}$ which is midway in the fusion depth. In figure 3 , the three sets of curves are for three different beam-on-times, namely, $t=0.01,0.05$, and $0.075 \mathrm{~s}$ corresponding to travel speeds of $2.5,0.5$ and $0.33 \mathrm{~cm} / \mathrm{s}$ respectively, but for the same given beam density $\left(W^{\prime}=2 \times 10^{10} \mathrm{~W} / \mathrm{m}^{2}\right)$. In figure 3 it can be seen that due to nearly equal lateral fusion-growths in the two metals, concentrations of the two elements in the central zone are almost equal. In addition, concentrations and distributions in a combination with metals having different thermophysical properties but equal meltings rendered by tuning the power sharing, will be described by the same figure. Moreover, in some metal combinations (close to figure 1(a)), the melt quantities will not be equal (due to unequal lateral fusion growths) even after proper power sharing for consistent fusion depth. The results for such fusion zones are illustrated in figure 4, a metal combination such as $\mathrm{Ni}-\mathrm{Cu}$ being an example in this case.

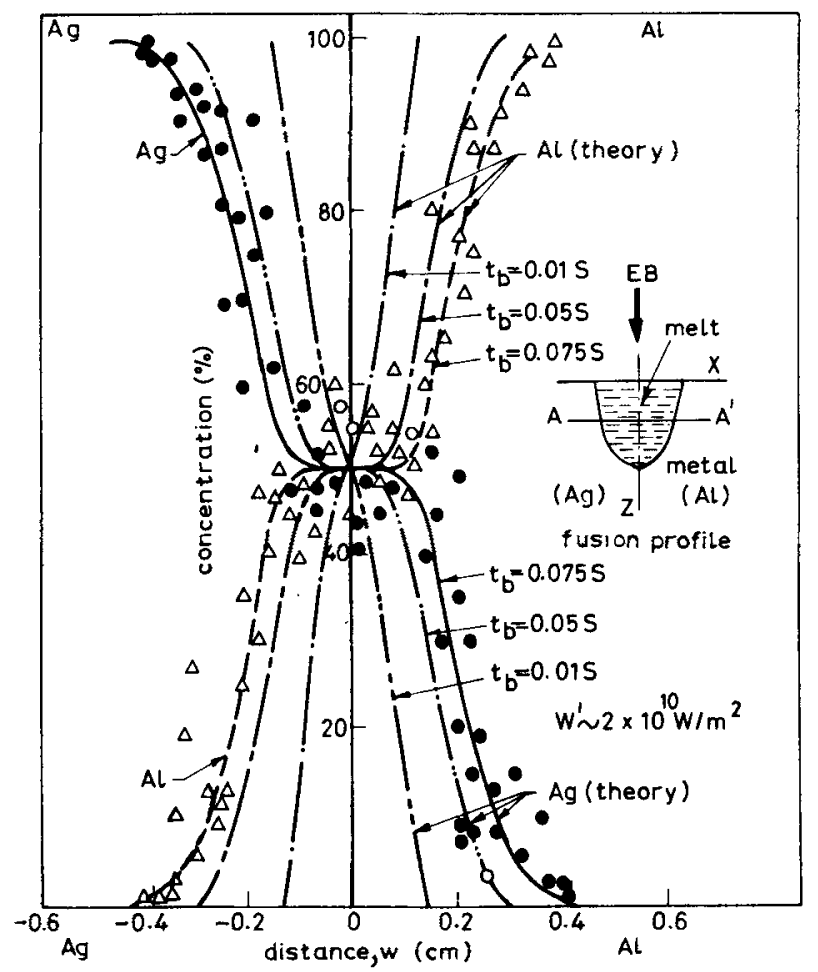

Figure 3. Mass concentrations in given section $\mathbf{A}-\mathbf{A}^{\prime}$ during transient fusion in the $\mathbf{A g}-\mathbf{A l}$ system and measured values $(\bullet) \mathrm{Ag}$ and $(\Delta) \mathrm{Al}$ corresponding to theory (- - ) and $(----)$ respectively 


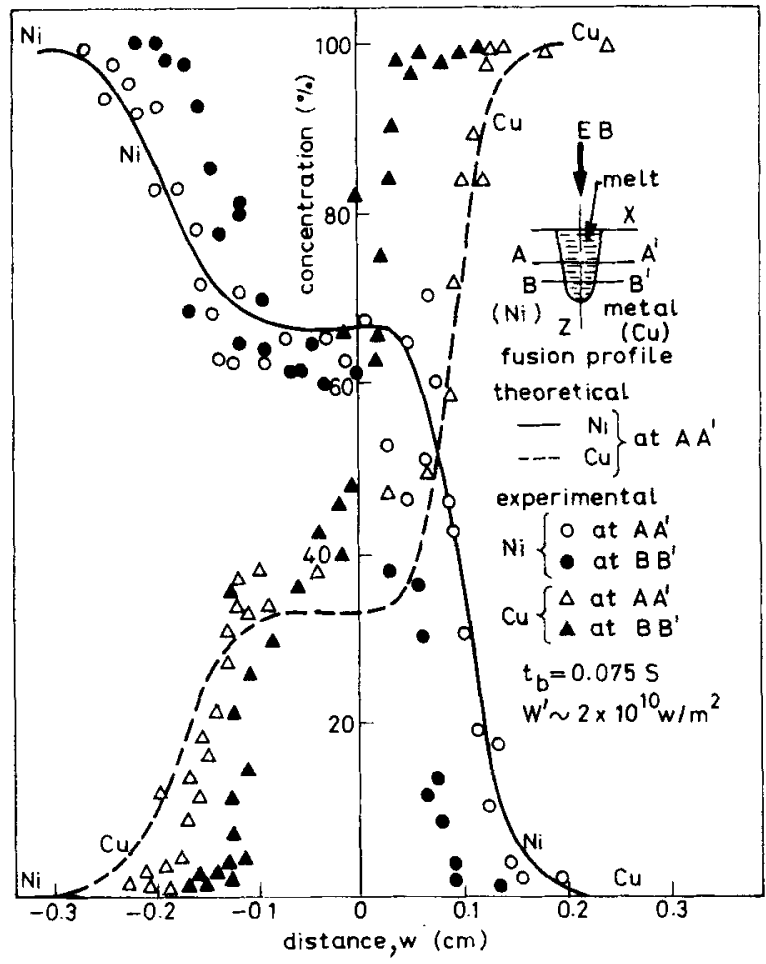

Figure 4. Typical mass concentrations in the fusion zone of a $\mathrm{Ni}-\mathrm{Cu}$ system, i.e. along given sections $A-A^{\prime}$ and $B-B^{\prime}$

\section{Experimental validation}

Experiments on fusing and joining of dissimilar metals have been conducted in the present study using an electron-beam facility. Electron beams of energy up to $150 \mathrm{keV}$ and powers of about a few $\mathrm{kW}$, have been focussed to high densities around $10^{10} \mathrm{~W} / \mathrm{m}^{2}$, and applied in the experiments (Vijayan and Rohatgi 1978). Using the optical-alignment system of the welding machine (Rohatgi 1983), the electron beam is located over the job to give the fractional beam as indicated in table 1 . In this manner different metal combinations have been butt-joined in the travel mode as applied in theory and studied here.

For analysing the mass concentrations in the fused zone, the welded samples have been cut along the $x-z$ plane, plane-finished and mirror-polished. The polished surface is then quantitatively analysed (Wells 1974) for mass concentrations using an electron probe microanalyser (EPMA), where the measurements are accurate to within $\pm 3 \%$.

From the results thus obtained for different samples, the measured results for $\mathrm{Al}-\mathrm{Ag}$ which has been fused earlier using a travel speed of $0.33 \mathrm{~cm} / \mathrm{s}$, are plotted in figure 3 , along with the theoretical curves $\left(t_{b}=0.075 \mathrm{~s}\right)$. It can be seen that the measured concentrations and their distributions agree reasonably well with the predicted values. Moreover, the measured plots indicate the presence of a uniformly-mixed zone as proposed in theory. 
The quantitative measurements from another joint, namely $\mathrm{Cu}-\mathrm{Ni}$, are presented in figure 4 along with the theoretical predictions at A-A'. The agreement between the measured and calculated results in this figure is also reasonably good. Apart from this,

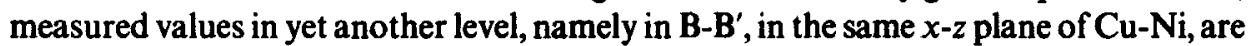
also plotted in figure 4. In this case, $B-B^{\prime}$ is chosen halfway between $A^{-} A^{\prime}$ and the root of the weld. It can be seen from figure 4 that the measurements at levels $A-A^{\prime}$ and $B-B^{\prime}$ reveal that the mass concentrations and their distributions in depth conform to the general pattern predicted by the present theory. Moreover, it can be seen from these plots that the width of the well-mixed central zone at $\mathbf{B}-\mathbf{B}^{\prime}$ is smaller than that at $\mathbf{A}-\mathbf{A}^{\prime}$. This, in fact, is precisely as expected in theory where fusion width $w$ at $\mathbf{B}^{-B^{\prime}}$ is smaller than at $\mathbf{A}-\mathbf{A}^{\prime}$.

The joint between $\mathrm{CU}-\mathrm{SS}$ is also chosen here for investigation, since it departs from a true binary system as one of the 'metals' involved in this case is an alloy (stainless steel). Here the effects on mass transport, due to the presence of a number of constituents in the melt, have been determined. The measured results from the fused joint of Cu-SS 304 are plotted in figure 5 along with the theoretical curves for different constituents. The agreement of the measurements with the predicted values is good in this figure. Moreover, the distributions of the concentration of the different constituents in the fusion zone conform to the general trend observed in the other examples cited here. Such distributions are brought about by rapid and forced mass transfer taking place due to agitation in the melt. Hence, the above observation leads to the important

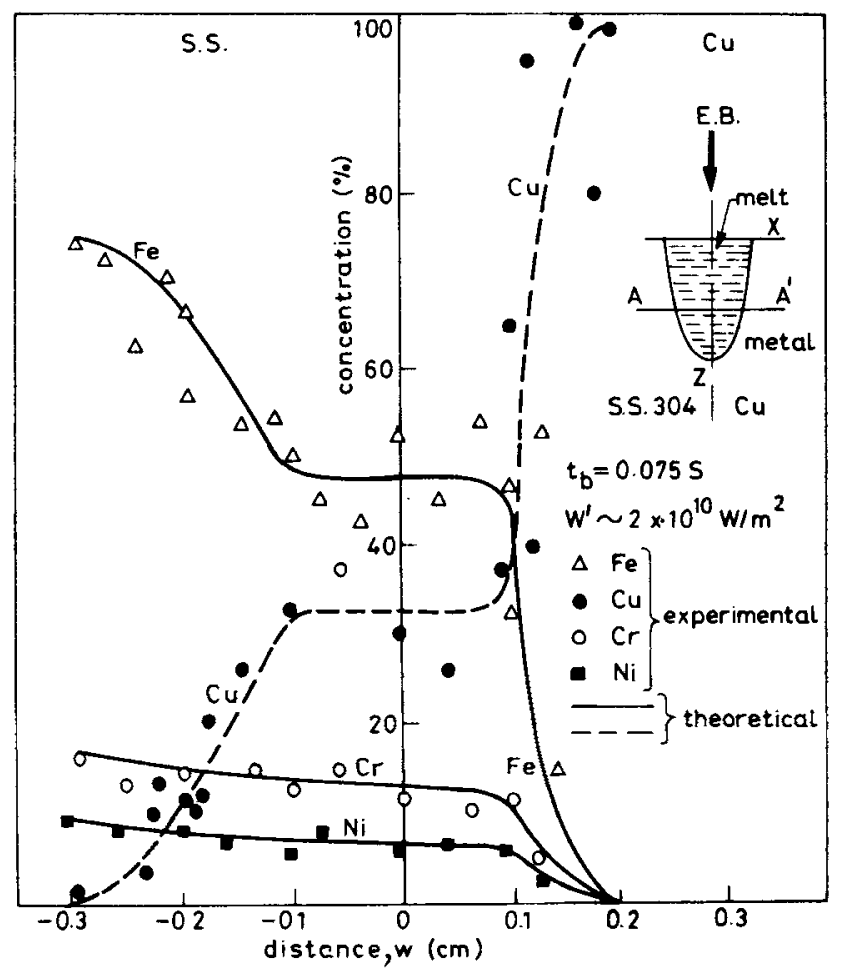

Figure 5. Measured mass concentrations in fusion profile and theoretical predictions in an SS $304-\mathrm{Cu}$ joint 
conclusion that the presence of a larger number of constituents in the melt does not alter the general trend of mass transfer during beam fusion. The effect of the constituents in this case is limited to their effect on the viscosities.

\section{Conclusions}

Physical and theoretical descriptions of beam fusion in dissimilar metal joints and intermixing of melts have been developed in the form of simple models. In order to render uniform fusion by the beam, power sharing in the two metals is determined through their geometries, heat contents and thermal conductivities. As far as mass transport is concerned, during beam fusion, it is brought about by violent agitation in the melt. In view of these agitations, mass transport coefficients more than two to three orders of magnitude higher than expected due to convection in the melt at prevailing temperature gradients, are predicted in the present investigations. Also, it is shown here that such turbulent conditions and resulting rapid intermixing produce a uniformlymixed central region in the fusion zone. The width of this region is dependent on the viscosity of the melt and the beam intensity.

Mass transfer during beam fusion is solved from a line-source solution of a divergence equation. In the time upto the solidification of the melt, mass transfer takes place through convection in a line source for the case of a stationary source. In the case of lateral source travel, convection subsequent to beam fusion is solved in a plane column of melt which is left behind by the beam but is yet to solidify. Using these models, mass concentrations and distributions in beam-fused binary joints have been theoretically predicted for various metal combinations. Quantitative measurements in experiments support the validity of the present models.

Acknowledgements-We are thankful to Shri C Ambasankaran for encouragement, to Shri K S Sivaramakrishnan, Dr P Rodrigues and Shri V Seetharaman for the analysis of samples. The critical comments offered by the referees were helpful in improving the quality of the paper.

\section{References}

Andrews J W, Atthey D R 1976 J. Phys. D: Appl. Phys. 9: 2181-2194

Beer S Z 1972 Liquid metals (New York: Marcel Dekker) p. 451

Cottrell A H 1964 Theoretical structural metallurgy, (UK: Camolet) p. 181

Crank J 1964 Mathematics of diffusion (London: Oxford University Press) p. 10

Geiger G H, Poirier D R 1973 Transport phenomena in metallurgy (Phillippines: Addison Wesley) p. 435

Klemens P G 1976 J. Appl. Phys. 47: 2165-2174

Miyazaki T, Taniguchi N 1972 Proceedings of the $V$ International Conference on electron and ion beam science and technology R Bakish (ed.) (Princeton: Electrochemical Society) p. 291

Rohatgi V K 1983 Development of electron beam welding machine, Final report, Aeronautics Research and Development Board, India

Rykalin N, Uglov A, Kokora A 1978 Laser machining and Welding, translated by O Glebov (Moscow: MIR)

Schwarz H 1964 J. Appl. Phys. 35: 2020-2029

Vijayan T 1982 Study of heat and mass transports in metals under electron-beam interactions, Ph.D. Thesis, University of Bombay, Bombay

Vijayan T, Rohatgi V K 1978 J. Phys. D: Appl. Phys. 11: 2157-2168 
Vijayan T, Rohatgi V K 1983 Physical behaviour of electron-beam fusion heat-transfer and deep penetration in metals, Int. J. Heat Mass Transfer (in press)

Weber C M 1972 Proceedings of the $V$ International Conference on electron and ion beam science and technology R Bakish (ed.) (Princeton: Electrochemical Society) p. 307

Wells O C 1974 Scanning electron microscopy (New York: McGraw Hill) p. 243

\section{A list of symbols}

\begin{tabular}{|c|c|}
\hline$A$ & metal $A$ \\
\hline$a$ & distance step $|\mathrm{m}|$ \\
\hline$a_{0}$ & acceleration in fusion-growth $\left|\mathrm{ms}^{-2}\right|$ \\
\hline$B$ & metal $B$ \\
\hline$b$ & $t$ parameter \\
\hline$b_{0}$ & constant \\
\hline$C, C_{0}, C_{A}, C_{B}$ & concentrations \\
\hline$C_{A}^{\prime}, C_{B}^{\prime}$ & concentrations in $w^{\prime}$ \\
\hline$C_{O A}, C_{0 B}$ & initial concentrations of $A, B$ \\
\hline$C_{A 1}$ & concentration of $A$ at a neighbouring point \\
\hline$c_{A}, c_{B}, c$ & specific heats of $A, B\left(\mathrm{~J} \mathrm{~kg}^{-1} \mathrm{~K}^{-1}\right)$ \\
\hline$D, D_{A}, D_{B}$ & coefficients of mass diffusion $\left(\mathrm{m}^{2} \mathrm{~s}^{-1}\right)$ \\
\hline$D_{s}$ & mass diffusivity in solid $\left(\mathrm{m}^{2} \mathrm{~s}^{-1}\right)$ (Geiger \& Poirier 1973) \\
\hline$D_{0}$ & frequency factor $\left(\mathrm{m}^{2} \mathrm{~s}^{-1}\right)$ \\
\hline$d$ & beam-spot width $(\mathrm{m})$ \\
\hline$E_{A}$ & activation energy $\left(\mathrm{J} \mathrm{mol}^{-1}\right)$ \\
\hline$h$ & fusion depth (m) \\
\hline$K, K_{A}, K_{B}$ & coefficients of thermal conductivity $\left(\mathrm{Jm}^{-1} \mathrm{~K}^{-1} \mathrm{~s}^{-1}\right)$ \\
\hline$K_{0}$ & thermal conductivity limit $\left(\mathrm{Jm}^{-1} \mathrm{~K}^{-1} \mathrm{~s}^{-1}\right)$ (Vijayan 1982) \\
\hline$L_{A}, L_{B}, L_{m}$ & latent heats of melting of $A, B\left(\mathrm{~J} \mathrm{~kg}^{-1}\right)$ \\
\hline$M_{l}$ & line source strength $\left(\mathrm{m}^{2}\right)$ \\
\hline$M_{p}$ & plane source strength (m) \\
\hline$Q_{A}, Q_{B}$ & heat inputs in $A, B(\mathrm{~J})$ \\
\hline$q$ & heat for melting $(\mathrm{J})$ \\
\hline$q_{m}$ & heat content in melt $(J)$ \\
\hline$q_{A}, q_{B}$ & melt quantities of $A, B\left(\mathrm{~m}^{3}\right)$ \\
\hline$R$ & universal gas constant $\left(\mathrm{J} \mathrm{K}^{-1} \mathrm{~mol}^{-1}\right)$ \\
\hline$T$ & temperature $(\mathbf{K})$ \\
\hline$T_{A}, T_{B}, T_{m}$ & melting points $(\mathrm{K})$ \\
\hline$T_{0}$ & ambient temperature (K) \\
\hline$t$ & time (s) \\
\hline$t_{1}$ & time for melt solidification in line source $(s)$ \\
\hline$t_{2}$ & time for melt solidification in plane source (s) \\
\hline$t_{b}$ & beam-on-time (s) \\
\hline$V$ & travel speed $\left(\mathrm{ms}^{-1}\right)$ \\
\hline $\begin{array}{l}V_{0} \\
W^{\prime}\end{array}$ & travel speed to give $y_{1}=w\left(\mathrm{~ms}^{-1}\right)$ \\
\hline$w$ & beam-power density $\left(\mathrm{Jm}^{-2} \mathrm{~s}^{-1}\right)$ \\
\hline$w$ & fusion width (m) \\
\hline
\end{tabular}




$$
\begin{aligned}
& w^{\prime} \\
& w_{0} \\
& x, y, z \\
& x_{0}, y_{0} \\
& y_{1} \\
& \alpha_{A}, \alpha_{B} \\
& \delta \\
& \rho_{A}, \rho_{B} \\
& \eta_{A}, \eta_{B} \\
& \eta_{T} \\
& \bar{\eta}
\end{aligned}
$$

width of uniform-mixed zone $(\mathrm{m})$

fusion width for maximum $w^{\prime}(\mathrm{m})$

distances $(\mathrm{m})$

initial distances $(\mathrm{m})$

length of melt column (m)

beam absorption coefficients in $A, B$

ratio of $q$ for $A, B$

densities of $A, B\left(\mathrm{~kg} \mathrm{~m}^{-3}\right)$

coefficients of viscosities for $A, B$ (Pa s)

coefficient of viscosity at temperature $T$ (Pa s) (Beer 1972)

average viscosity (Pa s) 